\title{
Efficient Sensor Placement for State Estimation in Structural Dynamics
}

Eric M. Hernandez ${ }^{\mathrm{a}, *}$

3 a School of Engineering, College of Engineering and Mathematical Sciences, University of Vermont, 33 Colchester Ave. 103 Votey Hall. ,VT, USA

\section{Abstract}

This paper derives a computationally efficient algorithm to determine optimal sequential sensor placement for state estimation in linear structural systems subjected to unmeasured excitations and noise contaminated measurements. The proposed algorithm is developed within the context of the Kalman filter and it minimizes the variance of the state estimate among all possible sequential sensor locations. The paper investigates the effects of measurement type, covariance matrix partition selection, spatial correlation of excitation and model selection on optimal sensor placement. The paper shows that the sequential approach reaches the optimal sensor placement as the number of sensor increases.

Keywords:

6 Kalman Filter, Sensor Placement, State Estimation, Stress Estimation, Structural Dynamics

\section{Introduction}

State estimation is a branch of control theory that deals with estimating hidden variables (the state) of a dynamical system based on a model and noise contaminated response measurements. This paper deals with state estimation in linear structural dynamics and in particular with the problem of determining optimal sensor locations such that the error in the estimated state or functions of the state is minimized. In this paper, the problem of optimal sensor placement will be defined as follows: given a linear model and covariance matrices for unmeasured loads and measurement noise, determine the particular sensor layout that minimizes the trace of the state error covariance of a user defined linear function of the state. A particular function of interest is the one that maps displacements at all degrees of freedom with the strains at points of interest. This function is significant in many applications, including fatigue monitoring of structures using global response measurements $[1,2]$.

One of the first documented efforts to apply state estimation in the context of structural dynamics was presented by Carmichael [3]. Subsequently, Waller and Schmidt [4,5] applied state estimation for monitoring stresses in critical and unaccessible locations in rotating machinery. They implemented the idea of a modal observer as a means to reduce the computational burden when working with large FE models. For systems in which the response can be spanned by a relatively small modal subspace, the estimator (or observer) can be implemented on a reduced order model that only contains the modes of interest. Wilde and Kozakiewicz [6] applied the Kalman filter to estimate the vibration of simple cylindrical cantilever structures partially submerged in water and subject to the interacting motion of the waves. In addition, the Kalman filter was also used to determine hydrodynamic force coefficients under certain steady state conditions. Hernandez and Bernal [7] proposed a robust observer to estimate the state of structural system with model uncertainties in mass, stiffness and(or) damping. More recently various researchers have proposed the use of state estimation as a means to track stress and strain fields in operational structures subjected to unmeasured excitations, the main objective being monitoring fatigue damage $[1,2,8,9,10,11]$.

The most common algorithm for state estimation is the Kalman filter [12, 13, 14]. In the Kalman filtering, the state of the system is estimated recursively at discrete time steps assuming a model of the system and the covariance matrices of the excitations and measurement noise. In the basic Kalman filter formulation it is assumed that the

${ }^{*}$ Corresponding author

Email address: eric.hernandez@uvm.edu (Eric M. Hernandez) 
excitations and measurement noise are Gaussian white noise sequences. Within the context of Kalman filtering, optimal sensor placement is achieved by selecting the state-to-output matrix such that the variance of the user-selected state function is minimized. Sometimes the trace of the state error covariance matrix is used as a global measure of optimality, however this is not always adequate if only a subset of states are of interest. For $m>1$ sensors, the problem of optimal sensor placement becomes a combinatorial problem, i.e. selecting among all possible combinations of placing $m$ sensors in $n$ possible locations. In most practical applications $n \gg m$, which makes the problem of optimal sensor placement computationally challenging.

This paper derives an efficient algorithm to determine sequential and near-optimal sensor placement which includes the possibility of spatio-temporal correlation in the excitations and measurement noise. The variance minimization approach has been employed by other researchers to determine optimal sensor placement in parameter identification and system identification of linear structural systems [16, 17, 18].

Most recently the problem of optimal sensor placement for state estimation and joint state-input estimation has been treated by Weickgenannt at al. [20], van den Linder et al.[19] and Zhang and Wang [21]. In [20] an optimal sensor location strategy was developed for an active shell structure. The approach was based on a dual-objective optimization, namely minimizing the number of sensors and a measure related to observability. The optimization was solved using a multi-objective simulated annealing algorithm. In [19], three approaches for optimal sensor placement were compared, namely: minimizing the static displacement estimation error, minimizing the inverse of the observability, and minimizing the dynamic displacement estimation error. All three approaches were found to provide similar error reduction, however their implementation cost was found to vary significantly, with the static approach being the least expensive by orders of magnitude. Each of the approaches was implemented and applied to a 2D model of the long-span New Carquinez Bridge in California. In [21] a sequential optimal sensor placement algorithm based on variance minimization was proposed. Finally, the use of observability as a criteria for optimal sensor placement for state estimation has been proposed and effectively implemented by other authors [19]. We compare the observability approach with the minimum variance approach and show under what conditions it may be more accurate.

The remainder of the paper is organized as follows, the first section presents the necessary theoretical background related to the systems of interest. This is followed by sections on observability, state estimation and Kalman filtering. The paper continues with a section describing optimal sensor placement in the context of the Kalman filter. This is followed by sections presenting numerical simulations that compare the effects of measurement types, covariance partition, spatio-temporal correlation of the input and observability versus minimum variance approach for optimal sensor placement. The paper ends with some final remarks and illustrative examples regarding the optimality and efficiency of the proposed sequential approach and conclusions.

\section{Models of Interest}

This paper is restricted to systems whose dynamic response can be accurately described by the following matrix ordinary differential equation

$$
\mathbf{M} \ddot{q}(t)+\mathbf{C}_{d} \dot{q}(t)+\mathbf{K} q(t)=\mathbf{b}_{1} u(t)+\mathbf{b}_{2} w(t)
$$

where $\mathbf{M}=\mathbf{M}^{T}>0 \in R^{N \times N}$ is the mass matrix, $\mathbf{C}_{d}=\mathbf{C}_{d}^{T}>0 \in R^{N \times N}$ is the damping matrix and $\mathbf{K}=\mathbf{K}^{T}>0 \in R^{N \times N}$ is the stiffness matrix. The vector $q(t) \in R^{N \times 1}$ is the displacement vector of the $N$ degrees of freedom, $\mathbf{b}_{1} \in R^{N \times r}$ defines the spatial distribution of the known excitation $u(t) \in R^{r \times 1}$ and $\mathbf{b}_{2} \in R^{N \times p}$ defines the spatial distribution of the unmeasured excitation $w(t) \in R^{p \times 1}$. By defining the state vector as $x(t)=\left[\begin{array}{ll}q^{T}(t) & \dot{q}^{T}(t)\end{array}\right]^{T} \in \mathbb{R}^{2 N \times 1}$, eq. 1 can be written in first order state-space form as

$$
\dot{x}(t)=\mathbf{A} x(t)+\mathbf{B}_{1} u(t)+\mathbf{B}_{2} w(t)
$$

where the matrices $\mathbf{A}, \mathbf{B}_{1}$ and $\mathbf{B}_{2}$ are

$$
\begin{gathered}
\mathbf{A}=\left[\begin{array}{cc}
\mathbf{0}_{N \times N} & \mathbf{I}_{N \times N} \\
-\mathbf{M}^{-1} \mathbf{K} & -\mathbf{M}^{-1} \mathbf{C}_{d}
\end{array}\right] \\
\mathbf{B}_{1}=\left[\begin{array}{c}
\mathbf{0}_{N \times r} \\
\mathbf{M}^{-1} \mathbf{b}_{1}
\end{array}\right] \quad \mathbf{B}_{2}=\left[\begin{array}{c}
\mathbf{0}_{N \times p} \\
\mathbf{M}^{-1} \mathbf{b}_{2}
\end{array}\right]
\end{gathered}
$$




\section{Measurement Models}

Measurements $y(t)$ of the dynamic response of the structure at discrete points will be represented as linear functions of the state with additive noise $v(t)$ as

$$
y(t)=\mathbf{C} x(t)+\mathbf{D}_{1} u(t)+\mathbf{D}_{2} w(t)+v(t)
$$

${ }_{5}$ For relative acceleration measurements $\mathbf{C}=\mathbf{c}_{2}\left[-\mathbf{M}^{-1} \mathbf{K} \quad-\mathbf{M}^{-1} \mathbf{C}_{d}\right], \mathbf{D}_{1}=\mathbf{c}_{2} \mathbf{M}^{-1} \mathbf{b}_{1}, \mathbf{D}_{2}=\mathbf{c}_{2} \mathbf{M}^{-1} \mathbf{b}_{2}$; for displacement measurements $\mathbf{C}=\left[\begin{array}{ll}\mathbf{c}_{2} & \mathbf{0}_{m \times N}\end{array}\right]$ and for velocity measurements $\mathbf{C}=\left[\begin{array}{ll}\mathbf{0}_{m \times N} & \mathbf{c}_{2}\end{array}\right]$. For displacement and velocity measurements $\mathbf{D}_{1}$ and $\mathbf{D}_{2}$ are zero matrices of appropriate dimensions. For displacement, velocity and acceleration measurements the output distribution matrix $\mathbf{c}_{2} \in R^{m \times N}$ is a Boolean matrix with every row having a single non-zero entry corresponding to the degree of freedom being measured and $m$ is the number of measurements.

In some situations strain measurements might be available. The corresponding $\mathbf{C}$ matrix for this case can be found by formulating a matrix $\mathbf{T}$ that maps the displacements at the degrees of freedom of the model with the strain measurement. To illustrate, consider a two node, elastic and planar beam-column element of length $L$ subjected only to nodal loads. The strain at any point in the element depends on the nodal displacement vector $q_{L}$ (using local coordinates).

$$
\varepsilon(x, z)=\mathbf{T} q_{L}
$$

where the vector $q_{L}$ is defined as $q_{L}^{T}=\left[\begin{array}{llllll}q_{1} & q_{2} & q_{3} & q_{4} & q_{5} & q_{6}\end{array}\right]$ (for subindices see Fig.1), $x$ is the distance along the element axis from the left node and $z$ is the height of the strain measurement from the neutral axis.

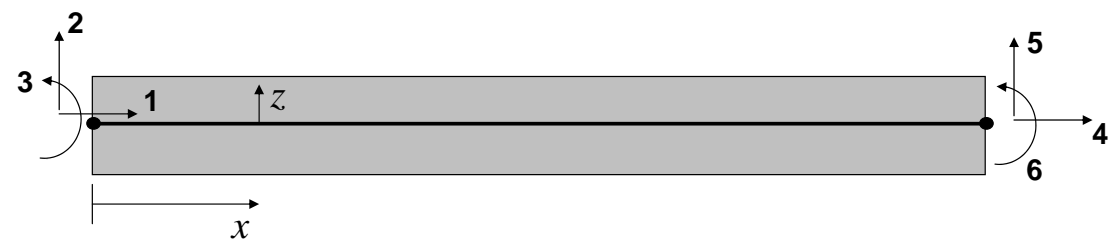

Figure 1: Nodal displacements in plane beam-column element

The transformation matrix $\mathbf{T}$ is given by

$$
\mathbf{T}=\left[\begin{array}{llllll}
-\frac{1}{L} & \frac{6 z}{L^{2}}-\frac{12 z}{L^{3}} x & \frac{4 z}{L}-\frac{6 z}{L^{2}} x & \frac{1}{L} & -\frac{6 z}{L^{2}}+\frac{12 z}{L^{3}} x & \frac{2 z}{L}-\frac{6 z}{L^{2}} x
\end{array}\right]
$$

Consequently the $\mathbf{C}$ matrix will contain the terms of the aforementioned $\mathbf{T}$ matrix multiplying the components of the state corresponding to the element(s) that have strain measurement(s).

Finally, the vector $v(t)$ in eq.5 is the measurement error. In the idealistic case where the model and the system coincide (at least within the frequency bandwidth of interest), the measurement error is usually modeled as a realization of a Gaussian random process with zero mean and known covariance matrix $\mathbf{R}(t)$. This tries to reproduce the phenomena of white noise experienced by measurement devices and sensors. In a more general formulation one can include specific models for measurement noise which try to account for the sensor dynamics, background noise and modeling errors.

\section{Observability}

A prerequisite for any state estimation to be successful is observability. A state $x\left(t_{o}\right)$ is observable if it can be determined from knowledge of the system matrices, inputs and outputs for $t>t_{o}$. If all possible states of a system are observable, then the system is globally observable.

A linear, finite dimensional and time invariant dynamical system such as the one described by eq. 2 with measurements given by eq.5 is globally observable iff the observability grammian $W_{o}$ defined by

$$
W_{o}\left(t_{f}\right)=\int_{0}^{t_{f}} e^{\mathbf{A}^{T} t} \mathbf{C}^{T} \mathbf{C} e^{\mathbf{A} t} d t>0
$$


is positive definite. A proof of this claim can be found by writing the output of a linear system as a convolution

$$
y(t)=\mathbf{C} e^{\mathbf{A} t} x_{o}+\mathbf{C} \int_{0}^{t} e^{\mathbf{A}(t-\tau)} \mathbf{B} u(\tau) d \tau
$$

or

$$
y_{r}(t)=y(t)-\mathbf{C} \int_{0}^{t} e^{\mathbf{A}(t-\tau)} \mathbf{B} u(\tau) d \tau=\mathbf{C} e^{\mathbf{A} t} x_{o}
$$

For the system to be completely observable any $x_{o} \neq 0 \in R^{N \times 1}$ must not lie in the null space of the operator $\mathbf{C} e^{\mathbf{A} t}$, which means that

$$
x_{o} \notin \mathscr{N}\left(\mathbf{C} e^{\mathbf{A}_{c} t}\right) \Longleftrightarrow\left\|\mathbf{C} e^{\mathbf{A} t} x_{o}\right\|^{2}>0
$$

By using the definition of the $l_{2}$ norm of a continuous operator it is found that

$$
\left\|\mathbf{C} e^{\mathbf{A} t} x_{o}\right\|_{2}^{2}=x_{o}^{T}\left(\int_{0}^{t} e^{\mathbf{A}^{T} \tau} \mathbf{C}^{T} \mathbf{C} e^{\mathbf{A} \tau} d \tau\right) x_{o}=x_{o}^{T} W_{o} x_{o}>0
$$

which can be achieved iff $W_{o}$ is positive definite. Notice that the criteria to decide whether a system is observable does not depend on the input. That is, if $W_{o}$ is positive definite then any initial condition can be reconstructed regardless of the input. This property is exclusive of linear systems.

Given that the system is observable, the initial state can be calculated as

$$
x_{o}=W_{o}^{-1} \int_{0}^{t_{f}} e^{\mathbf{A}^{T} t} \mathbf{C}^{T} y_{r}(t) d t \quad t_{f}<t
$$

where $y_{r}(t)$ has been previously defined in eq.10 and the invertibility of $W_{o}$ is guaranteed given its positive definiteness. In practical situations, the degradation in the estimate of the initial state in the presence of measurement noise needs to be investigated. Let's denote $x_{o}$ as the true initial state, $\xi_{o}$ as the estimated state in the presence of output noise $v$. Then the error in the estimated state $e_{o}=x_{o}-\xi_{o}$ is given by

$$
e_{o}=W_{o}^{-1} \int_{0}^{t_{f}} e^{\mathbf{A}^{T} t} \mathbf{C}^{T} v(t) d t
$$

The detrimental effects of measurement noise in the estimation of the state is governed mainly by the condition number of the observability grammian. It is possible to use the condition number of the observability grammian as a criterion for optimal sensor placement specifically selecting the measurement location such that the condition number of the observability grammian is minimized. As can be ascertained from the formulas above, that approach does not account for the loading conditions, resulting in a possibly sub-optimal sensor placement. This aspect will be illustrated within the context of simulations in Section 7 of the paper.

\section{Kalman-Bucy Filter with Colored Excitation}

This paper uses the Kalman filter as a means to determine optimal sensor placement. This section describes the main equations to implement the Kalman filter. The Kalman filter was originally formulated as a discrete estimator, however for the purposes of this paper, it will be convenient to manipulate the Kalman filter as if measurements were available continuously. This will facilitate some of the derivations to follow and it is consistent with most practical situations in structural dynamics where the sampling frequency is high with respect to the governing dynamics of the system.

The continuous time formulation of the Kalman filter is known as the Kalman-Bucy filter. The Kalman-Bucy filter is an estimator of the form

$$
\dot{\hat{x}}(t)=\mathbf{A} \hat{x}(t)+\mathbf{K}(t)\left(y(t)-\mathbf{C} \hat{x}(t)-\mathbf{D}_{1} u(t)\right)
$$


where $\hat{x}(t)$ is the state estimate and $\mathbf{K}(t)$ is the Kalman gain. In the Kalman-Bucy filter formulation it is assumed that the unmeasured excitations $w(t)$ and measurement error $v(t)$ are realizations of Gaussian random processes with zero mean and covariance matrices $\mathbf{Q}(t)$ and $\mathbf{R}(t)$ respectively. The objective function is the expected value of the Euclidean norm of the state error vector, namely $\operatorname{Tr}(\mathbf{P}(t))$, where $\mathbf{P}(t)$ is the state error covariance given by the solution of the following Riccati equation

$$
\dot{\mathbf{P}}(t)=\mathbf{A} \mathbf{P}(t)+\mathbf{P}(t) \mathbf{A}^{T}-\mathbf{K}(t) \mathbf{R}(t) \mathbf{K}(t)^{T}+\mathbf{Q}(t)
$$

where $\mathbf{K}(t)$ is given by

$$
\mathbf{K}(t)=\mathbf{P}(t) \mathbf{C}^{T} \mathbf{R}(t)^{-1}
$$

It can be shown that given the assumptions, the previous equation is the optimal choice for the gain matrix in the sense that they minimize a quadratic state error objective function $[14,13]$.

\subsection{Spatial and Temporal Correlation}

Within the context of the Kalman-Bucy filter, modeling temporal and spatial correlations in the excitation forces can be achieved by assuming the excitation vector $w(t)$ is the output of an auxiliary linear system of the form

$$
\begin{aligned}
& \dot{\xi}(t)=\mathbf{E} \xi(t)+\mathbf{G} n(t) \\
& w(t)=\mathbf{H} \xi(t)+\mathbf{L} n(t)
\end{aligned}
$$

where $n(t)$ is typically selected as a zero mean, white, Gaussian random process and the matrices $\mathbf{E}, \mathbf{G}, \mathbf{H}$ and $\mathbf{L}$ are strategically selected in order to achieve the desired temporal and spatial correlation. Determining suitable dimensions and values for these matrices is not a trivial task and it is application dependent. Needless to say that not all conceivable or desirable correlations can be modeled using this approach, however if it works, then the covariance of $w(t)$ can be related to the covariance of $n(t)$ as follows

$$
\begin{gathered}
\dot{\Xi}(t)=\mathbf{E} \Xi(t) \mathbf{E}^{T}+\mathbf{G} N(t) \mathbf{G}^{T} \\
\mathbf{Q}(t)=\mathbf{H} \Xi(t) \mathbf{H}+\mathbf{L} N(t) \mathbf{L}^{T}
\end{gathered}
$$

where $\mathbf{Q}(t)$ is the target covariance of $w(t), \Xi(t)$ is the covariance matrix of the auxiliary state vector $\xi(t)$ and $N(t)$ is the covariance of the noise vector $n(t)$. As mentioned previously the objective is to select the matrices $\mathbf{E}, \mathbf{G}, \mathbf{H}, \mathbf{L}$ and $N(t)$ to obtain the desired $\mathbf{Q}(t)$.

The resulting combined linear system, which incorporates the spatial and temporal correlation of the excitation is given by

$$
\begin{gathered}
{\left[\begin{array}{c}
\dot{x}(t) \\
\dot{\xi}(t)
\end{array}\right]=\left[\begin{array}{cc}
\mathbf{A} & \mathbf{B}_{2} \mathbf{H} \\
\mathbf{0}_{n \times 2 N} & \mathbf{E}
\end{array}\right]\left[\begin{array}{l}
x(t) \\
\xi(t)
\end{array}\right]+\left[\begin{array}{c}
\mathbf{B}_{1} \\
\mathbf{0}_{n \times r}
\end{array}\right] u(t)+\left[\begin{array}{c}
\mathbf{B}_{2} \mathbf{L} \\
\mathbf{G}
\end{array}\right] n(t)} \\
y(t)=\left[\begin{array}{cc}
\mathbf{C} & \mathbf{D}_{2} \mathbf{H}
\end{array}\right]\left[\begin{array}{l}
x(t) \\
\xi(t)
\end{array}\right]+\mathbf{D}_{1} u(t)+\mathbf{D}_{2} \mathbf{L} n(t)+v(t)
\end{gathered}
$$

The coupled system of equations can then be written as

$$
\begin{gathered}
\dot{z}(t)=\mathbf{A}_{c} z(t)+\mathbf{B}_{c 1} u(t)+\mathbf{B}_{c 2} n(t) \\
y(t)=\mathbf{C}_{c} z(t)+\mathbf{D}_{1} u(t)+\mathbf{D}_{2} \mathbf{L} n(t)+v(t)
\end{gathered}
$$

where $z(t)=\left[x(t)^{T} \xi(t)^{T}\right]^{T}$, the subscript $c$ indicates the matrices of the coupled systems and the definition of the coupled system matrices can be inferred from the previous equations. 


\section{Optimal Sequential Sensor Placement Using Minimum Variance}

In order to achieve a well-posed optimal sensor placement problem it is necessary to define an optimality criterion. In the context of the Kalman filter, this is achieved by selecting sensor types and locations such that the variance of a user-defined linear function of the state given by

$$
g(t)=\mathbf{S} x(t)
$$

is minimized, where $\mathbf{S}$ is a matrix of appropriate dimensions. This linear function typically corresponds to a particular set of internal forces, stresses and(or) strains that need to be monitored during the operation of the structure and which can not be measured directly. In mathematical terms, optimal sensor placement involves selecting the terms of the matrix $\mathbf{C}$ such that they meet some physical constraints and at the same time satisfy

$$
\mathbf{C}: \min \left(\operatorname{Tr}\left(\mathbf{S P}(t) \mathbf{S}^{T}\right)\right)
$$

In order to apply the previous algorithm two aspects are important: (i) the combinatorial problem of sensor location selection and (ii) the effect of spatio-temporal correlation of the loading. In the combinatorial sense, for a model with $n$ possible sensor locations and $m$ measurements, one must theoretically evaluate $\left(\begin{array}{c}n \\ m\end{array}\right)=\frac{n !}{m !(n-m) !}$ possible combinations in order to determine the optimal sensor placement. In each case one would evaluate eq.16 and 27 to determine which sensor location minimizes the trace of the desired covariance matrix. In the special case were $\mathbf{Q}=\mathbf{Q}_{o}$ and $\mathbf{R}=\mathbf{R}_{o} \forall t$ one can simplify the problem by looking at the steady-state solution of the Riccati equation $\left(\mathbf{P}_{s}\right)$, which can be obtained by setting $\dot{\mathbf{P}}=0$ and solving

$$
\mathbf{0}=\mathbf{A} \mathbf{P}_{s}+\mathbf{P}_{s} \mathbf{A}^{T}-\mathbf{P}_{s} \mathbf{C}^{T} \mathbf{R}_{o}^{-1} \mathbf{C}^{T} \mathbf{P}_{s}+\mathbf{Q}_{o}
$$

For each sensor arrangement one can compute the trace of $\mathbf{S P}_{s} \mathbf{S}^{T}$ and determine which of the $n$-choose- $m$ sensor arrangements results in the smallest trace. This procedure is cumbersome and time consuming, specially as the number of degrees of freedom increases.

In the proposed algorithm, optimal placement of $m$ sensors will be achieved by sequentially finding the optimal location of a single additional sensor given that the previous sensors have been placed optimally. To illustrate consider, without loss of generality, the case where $\mathbf{S}=\mathbf{I}$ and the steady-state solution to the state error covariance equation with output distribution matrix $\mathbf{C}_{1}$

$$
\mathbf{0}=\mathbf{A} \mathbf{P}_{1}+\mathbf{P}_{1} \mathbf{A}^{T}-\mathbf{P}_{1} \mathbf{C}_{1}^{T} \mathbf{R}_{o}^{-1} \mathbf{C}_{1}^{T} \mathbf{P}_{1}+\mathbf{Q}_{o}
$$

Let's assume that the selected $\mathbf{C}_{1}$ is optimal and that the resulting $\mathbf{P}_{1}$ achieves the minimal trace. Adding a new row to the $\mathbf{C}_{1}$ matrix, i.e. adding one measurement, results in a new steady-state Riccati equation with solution $\mathbf{P}_{2}$

$$
\mathbf{0}=\mathbf{A} \mathbf{P}_{2}+\mathbf{P}_{2} \mathbf{A}^{T}-\mathbf{P}_{2}\left[\begin{array}{ll}
\mathbf{C}_{1}^{T} & \mathbf{C}_{2}^{T}
\end{array}\right]\left[\begin{array}{cc}
\mathbf{R}_{1}^{-1} & 0 \\
0 & \mathbf{R}_{2}^{-1}
\end{array}\right]\left[\begin{array}{l}
\mathbf{C}_{1} \\
\mathbf{C}_{2}
\end{array}\right] \mathbf{P}_{2}+\mathbf{Q}_{o}
$$

$$
\mathbf{0}=\mathbf{A} \mathbf{P}_{2}+\mathbf{P}_{2} \mathbf{A}^{T}-\mathbf{P}_{2}\left(\mathbf{C}_{1}^{T} \mathbf{R}_{1}^{-1} \mathbf{C}_{1}+\mathbf{C}_{2}^{T} \mathbf{R}_{2}^{-1} \mathbf{C}_{2}\right) \mathbf{P}_{2}+\mathbf{Q}_{o}
$$

By substituting $\mathbf{P}_{2}=\mathbf{P}_{1}+\Delta \mathbf{P}$ in the previous equation and subtracting eq. 29 from eq. 31 we find after some algebra and rearrangement of terms that

$$
\mathbf{0}=\left(\mathbf{A}-\mathbf{P}_{1} \mathbf{C}^{T} \mathbf{R}^{-1} \mathbf{C}\right) \Delta \mathbf{P}+\Delta \mathbf{P}\left(\mathbf{A}^{T}-\mathbf{P}_{1} \mathbf{C}^{T} \mathbf{R}^{-1} \mathbf{C}\right)-\Delta \mathbf{P} \mathbf{C}^{T} \mathbf{R}^{-1} \mathbf{C} \Delta \mathbf{P}-\mathbf{P}_{1} \mathbf{C}_{2}^{T} \mathbf{R}_{2}^{-1} \mathbf{C}_{2} \mathbf{P}_{1}
$$

where

$$
\mathbf{C}=\left[\begin{array}{l}
\mathbf{C}_{1} \\
\mathbf{C}_{2}
\end{array}\right] \quad \mathbf{R}=\left[\begin{array}{cc}
\mathbf{R}_{1} & 0 \\
0 & \mathbf{R}_{2}
\end{array}\right]
$$

Eq.32 proves that it is possible to compute the change (reduction) in the covariance matrix $\Delta \mathbf{P}$ induced by adding a new measurement based on the covariance matrix $\mathbf{P}_{1}$ prior to including the new measurement. It fact, this equation 
is itself a Riccati equation. The only restriction to this procedure is that the measurement noise in the added sensor be uncorrelated with the measurement noise in all the initial sensors.

The previous derivations shows that by operating sequentially one only needs to examine $\sum_{j=0}^{m-1}(n-j)$ combinations and not $\left(\begin{array}{c}n \\ m\end{array}\right)=\frac{n !}{m !(n-m) !}$. However, it is not possible to prove that the optimal sequential sensor placement is absolutely optimal. The discrepancy between seqential and exhaustive sensor placement will be examined in Section 8 of the paper.

\section{Simulation Results}

This section presents simulation results aimed at illustrating the effects of the following factors on optimal sensor placement: (i) measurement type (ii) load correlation, (iii) partition of covariance matrix for objective function and (iv) model selection. We also compare the proposed minimum variance approach with the observability criterion for optimal sensor placement.

All simulations will be carried out on a 30-degree of freedom cantilever shear-beam with uniform discrete masses and spring stiffnesses. The mass of every degree of freedom is $m=1 \mathrm{Kg}$ and each spring has a stiffness of $k=$ $5000 \mathrm{~N} / \mathrm{m}$. The damping is classical with $5 \%$ of critical damping for every mode. Fig.2 depicts the first four modes shapes and their corresponding frequencies resulting from a modal analysis of the 30-degree of freedom cantilever shear-beam.
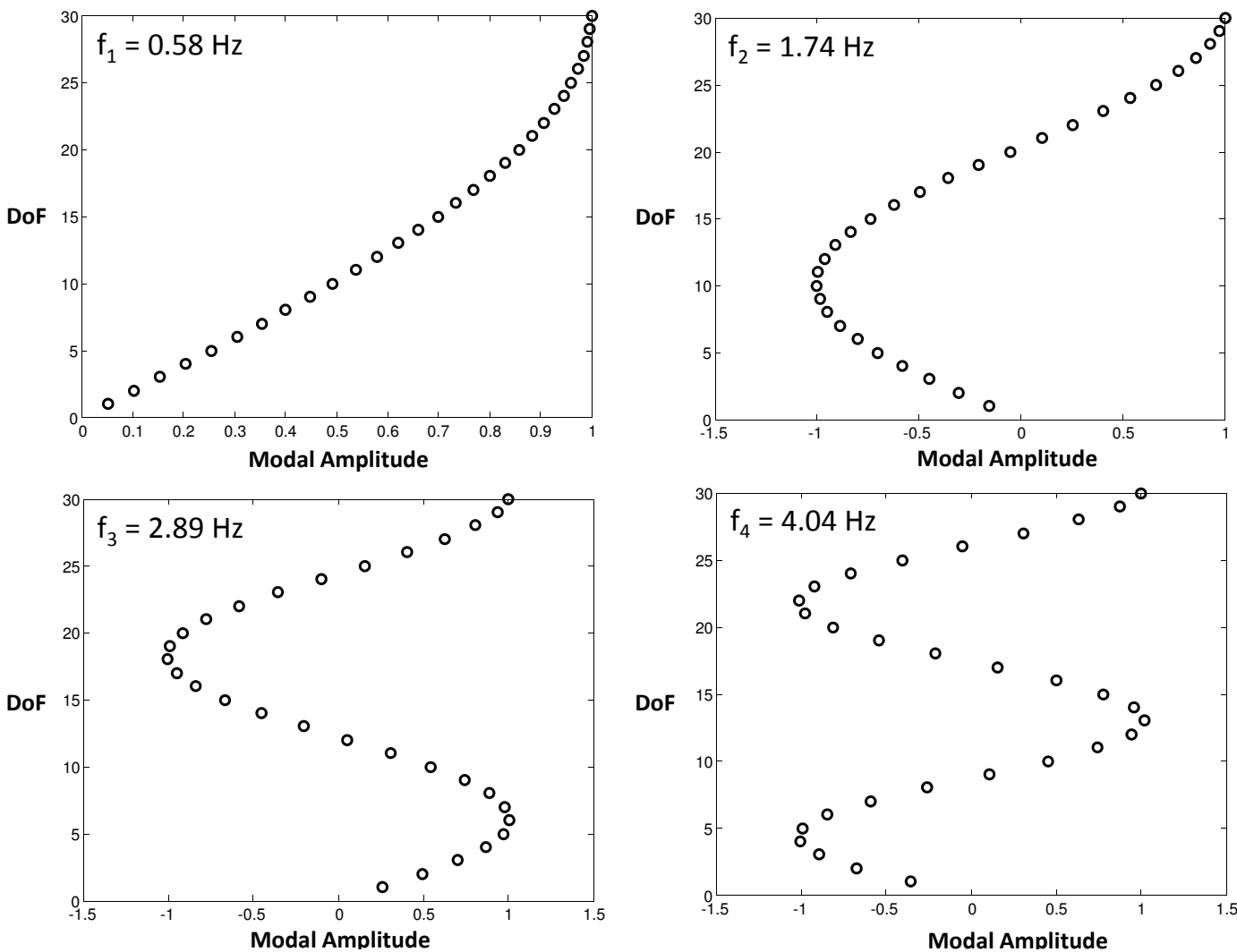

Figure 2: First four mode shapes and their corresponding natural frequencies computed for 30-DoF vertical shear-beam structure considered in the simulations. 


\subsection{Effect of Measurement Type}

To investigate the effects of measurement type on optimal sequential sensor placement we consider the case of uncorrelated loads (in space and time) with zero mean and $\mathbf{Q}=\mathbf{I}_{N \times N}$. The sensor placement was determined by implementing the incremental algorithm presented in section 6 . The resulting sensor placement for the first 15 sensors is presented in Table 1. The results are shown for displacement, velocity and acceleration measurements. The variance of the measurement noise (assumed to be a zero-mean Gaussian random process) was selected such that the noise-to-signal ratio (in an RMS sense) at the top mass (DoF 30) is 0.05. This results in the following measurement noise spectral density matrices $\mathbf{R}_{\text {dis }}=(0.0004 m)^{2} \mathbf{I}_{m \times m}$ for displacement measurements, $\mathbf{R}_{v e l}=(0.0027 \mathrm{~m} / \mathrm{s})^{2} \mathbf{I}_{m \times m}$ for velocity measurements and $\mathbf{R}_{a c c}=\left(0.09 \mathrm{~m} / \mathrm{s}^{2}\right)^{2} \mathbf{I}_{m \times m}$ for acceleration measurements.

Fig. 3 shows the reduction in the trace of the state error covariance matrix as a function of sequentially and optimally increasing the number of sensors as presented in Table 1. The results illustrate various important trends: (i) The type of measurement makes a significant difference in the optimal sensor placement, (ii) displacement and velocity measurements are more effective than acceleration measurements, and (iii) increasing the number of sensors yields diminishing returns in reducing the state estimation uncertainty.

Table 1 also presents a comparison between the minimum variance approach and the observability criterion. The order of sensor placement in the observability criterion was determined based on the condition number of the observability grammian. We placed one sensor at a time in such a way that the selected position (along with the previously placed sensors) results in the minimum condition number of the resulting observability matrix.

Table 1: Optimal sequential sensor placement for a uniform shear-beam subjected to uncorrelated loads. Three different types of measurements and the full trace of $\mathbf{P}$ were considered. The optimal sensor placement based on observability is also presented.

\begin{tabular}{|l||c|c|c|c|c|c|c|c|c|c|c|c|c|c|c|}
\hline \multicolumn{1}{|c||}{} & \multicolumn{10}{c|}{ Optimal Sequential Sensor Placement Sequence } \\
\hline Meas. Type & $\mathbf{1}$ & $\mathbf{2}$ & $\mathbf{3}$ & $\mathbf{4}$ & $\mathbf{5}$ & $\mathbf{6}$ & $\mathbf{7}$ & $\mathbf{8}$ & $\mathbf{9}$ & $\mathbf{1 0}$ & $\mathbf{1 1}$ & $\mathbf{1 2}$ & $\mathbf{1 3}$ & $\mathbf{1 4}$ & $\mathbf{1 5}$ \\
\hline Displacement & 30 & 3 & 17 & 14 & 23 & 8 & 21 & 10 & 26 & 5 & 12 & 19 & 1 & 28 & 16 \\
\hline Velocity & 30 & 5 & 17 & 12 & 21 & 8 & 24 & 3 & 15 & 26 & 10 & 20 & 2 & 27 & 13 \\
\hline Acceleration & 30 & 15 & 4 & 23 & 27 & 9 & 20 & 1 & 17 & 26 & 6 & 12 & 29 & 2 & 22 \\
\hline Observability & 26 & 15 & 5 & 11 & 30 & 23 & 20 & 8 & 29 & 18 & 13 & 3 & 1 & 24 & 6 \\
\hline
\end{tabular}

\subsection{Effect of Covariance Matrix Partition Selection}

In the previous section results for optimal sequential sensor placement based on minimizing the full state error covariance matrix $\mathbf{P}$ were presented. However, by examining the internal partitions of $\mathbf{P}$ in the case where the state is partitioned as given by eq. 2 one finds that

$$
\mathbf{P}=\left[\begin{array}{ll}
\mathbf{P}_{d d} & \mathbf{P}_{d v} \\
\mathbf{P}_{d v}^{T} & \mathbf{P}_{v v}
\end{array}\right]
$$

where the subindex $d$ stands for displacement and the subindex $v$ stands for velocity. As one can see; three blocks define $\mathbf{P}$, namely the displacement error covariance block $\mathbf{P}_{d d}$, the joint displacement and velocity covariance block $\mathbf{P}_{d v}$ and the velocity error covariance block $\mathbf{P}_{v v}$. Two important aspects are: (i) these blocks have different units, with the velocity error covariance block having the largest numerical values, and thus when the trace of the complete matrix $\mathbf{P}$ is computed, the velocity error governs the numerical value of the trace, (ii) if one is interested in applying state estimation for stress and strain estimation purposes as in $[1,10]$, then it is better to determine optimal sensor placement based on $\operatorname{Tr}\left(\mathbf{P}_{d d}\right)$ and not $\operatorname{Tr}(\mathbf{P})$. This because the estimates of strain and stress are linear functions of the displacement estimates and thus minimizing the error in those in more relevant.

Table 2 shows the sensor placement for the first 15 sensors if only the displacement portion of the state error covariance matrix is used. Similarly to the previous section, uncorrelated loads (in space and time) with zero mean and unit variance applied simultaneously at all degrees of freedom are considered. The optimal sensor placement was determined by implementing the incremental algorithm presented in section 6 . The table is shown for displacement, velocity and acceleration measurements. 


\section{$\operatorname{Tr}(\mathbf{P})$}

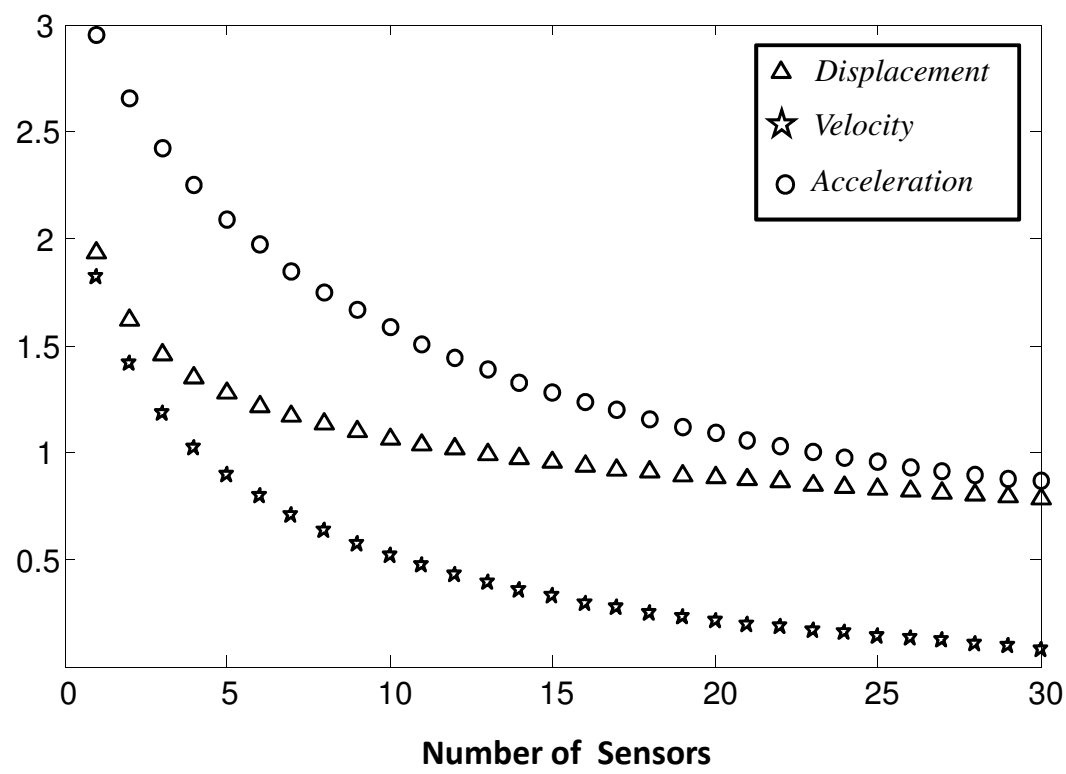

Figure 3: Reduction in the trace of the state error covariance matrix $(\mathbf{P})$ as a function of the number of sensors. Results are shown for displacement, velocity and acceleration measurements in the case of spatially uncorrelated loads.

Fig.4 shows the reduction in the trace of $\mathbf{P}_{d d}$ as a function of increasing the number of sensors. In comparison with Fig.3 the reader can notice three important differences: (i) the units of the trace of the displacement partition of the covariance matrix are significantly lower that the units of the complete trace of $\mathbf{P}$, this corroborates one of the previous argument, (ii) The optimal sensor placement has now changed in comparison with the case where the complete trace of $\mathbf{P}$ is used, and finally (iii) the relative difference between acceleration and displacement and velocity measurements is accentuated.

Table 2: Optimal sequential sensor placement for a uniform shear-beam subjected to uncorrelated loads. Three different types of measurements and the trace of $\mathbf{P}_{d d}$ were considered.

\begin{tabular}{|l||c|c|c|c|c|c|c|c|c|c|c|c|c|c|c|}
\hline \multicolumn{1}{|c||}{} & \multicolumn{11}{|c|}{ Optimal Sequential Sensor Placement Sequence } \\
\hline Meas. Type & $\mathbf{1}$ & $\mathbf{2}$ & $\mathbf{3}$ & $\mathbf{4}$ & $\mathbf{5}$ & $\mathbf{6}$ & $\mathbf{7}$ & $\mathbf{8}$ & $\mathbf{9}$ & $\mathbf{1 0}$ & $\mathbf{1 1}$ & $\mathbf{1 2}$ & $\mathbf{1 3}$ & $\mathbf{1 4}$ & $\mathbf{1 5}$ \\
\hline Displacement & 26 & 11 & 17 & 6 & 30 & 21 & 3 & 14 & 23 & 8 & 19 & 28 & 4 & 13 & 9 \\
\hline Velocity & 27 & 12 & 18 & 8 & 24 & 30 & 5 & 20 & 15 & 26 & 22 & 3 & 29 & 11 & 28 \\
\hline Acceleration & 30 & 28 & 29 & 1 & 26 & 2 & 27 & 24 & 3 & 25 & 4 & 23 & 22 & 5 & 21 \\
\hline
\end{tabular}

\subsection{Effect of Spatial Correlation of Loads}

This section the effect of spatial correlation of the loading will be examined. In the case of perfectly correlated loads applied at all degrees of freedom with zero mean and unit variance, it is found that implementation of the incremental algorithm presented in section 6 yields an optimal sensor placement as shown in Table 3 (contrast this with the case of uncorrelated loads shown in Table 2). The variance of the measurement noise (assumed to be a zero-mean Gaussian random process) was selected such that the noise-to-signal ratio (in an RMS sense) at the top mass is 0.05. This results in the following measurement noise spectral density matrices $\mathbf{R}_{\text {dis }}=(0.002)^{2} \mathbf{I}_{m \times m}$ for displacement measurements, $\mathbf{R}_{v e l}=(0.008)^{2} \mathbf{I}_{m \times m}$ for velocity measurements and $\mathbf{R}_{a c c}=(0.04)^{2} \mathbf{I}_{m \times m}$ for acceleration measurements. 




Figure 4: Reduction in the trace of $\mathbf{P}_{d d}$ as a function of the number of sensors. Results are shown for displacement, velocity and acceleration measurements in the case of spatially uncorrelated loads.

Fig.5 shows the reduction in the trace of $\mathbf{P}_{d d}$ as a function of increasing the number of sensors. Similar conclusions to the previous cases can be drawn regarding the effectiveness of measurement types and the diminishing returns of additional measurements.

Table 3: Optimal sequential sensor placement for a uniform shear-beam subjected to fully correlated loads. Three different types of measurements and the trace of $\mathbf{P}_{d d}$ were considered.

\begin{tabular}{|c|c|c|c|c|c|c|c|c|c|c|c|c|c|c|c|}
\hline & \multicolumn{15}{|c|}{ Optimal Sequential Sensor Placement $\left(\right.$ based on $\left.\operatorname{Tr}\left(\mathbf{P}_{d i s}\right)\right)$} \\
\hline Mea & 1 & 2 & 3 & 4 & 5 & 6 & 7 & 8 & 9 & 10 & 11 & 12 & 13 & 14 & 15 \\
\hline Dis & 15 & 26 & 7 & 8 & 21 & 29 & 6 & 14 & 5 & 20 & 28 & 12 & 4 & 19 & 24 \\
\hline Velor & 28 & 27 & 30 & 29 & 26 & 25 & 24 & 23 & 22 & 21 & 20 & 19 & 18 & 17 & 16 \\
\hline Acceleration & 25 & & 30 & 2 & 29 & 3 & 28 & 4 & 27 & 26 & 5 & 24 & 6 & 23 & 22 \\
\hline
\end{tabular}

From these results it appears that if the load is uncorrelated spatially, displacement measurements appear to be more efficient, while when loads were perfectly correlated, acceleration measurements are more effective. This conclusion is clearly only applicable to the cantilever shear-beam structure that was considered in this example.

\subsection{Effect of Model Selection}

This section presents a numerical investigation into the effect of model selection. We compare results from a cantilever shear-beam model versus a moment-frame model. The shear-beam model is as described in section 7 . The moment-frame model is composed of two columns and one beam per floor. The height of each floor is $2 \mathrm{~m}$ and the bay length $1 \mathrm{~m}$. The moment of inertia of the beams and columns is the same and equal to $78.62 \times 10^{-4} \mathrm{~m}^{4}$. The load covariance matrix is given by $\mathbf{Q}=\mathbf{I}_{N \times N}$. Measurements are assumed to be displacements and the variance of the measurement noise (assumed to be a zero-mean Gaussian random process) was selected as $\mathbf{R}_{\text {dis }}=(0.0004 m)^{2} \mathbf{I}_{m \times m}$. 


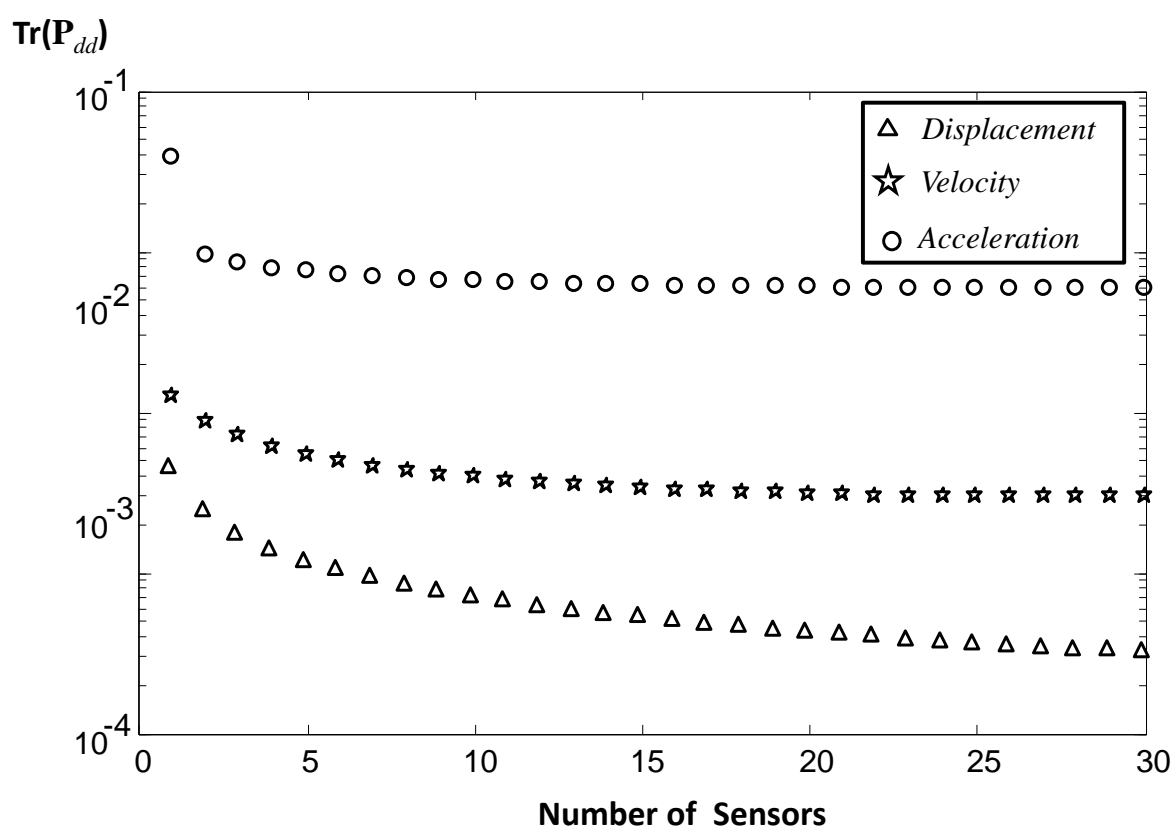

Figure 5: Reduction in the trace of $\mathbf{P}_{d d}$ as a function of the number of sensors. Results are shown for displacement, velocity and acceleration measurements in the case of spatially correlated loads.

Both models are formulated such that the fundamental period of vibration is the same $\left(f_{n}=0.58 \mathrm{~Hz}\right)$. The damping is defined as classical with 0.05 of critical for every mode. For this case optimal sensor placement was defined by minimizing the trace of the displacement partition of the state error covariance matrix.

Table 4 shows the results of the optimal sensor placement for the first 15 sensors. As can be seen the sensor placement for the first three sensors is almost the same for both models. After that point the optimal sensor placement differs. Fig.6 depicts the reduction in the trace of the displacement portion of the state error covariance matrix as a function of the number of sensors (per Table 4). It can be seen that the estimated state error covariance is a bit larger for the shear building model than from the frame building model.

Table 4: Comparison of optimal sequential sensor placement for different types of models, displacement measurements and minimization of the trace of $P_{d d}$

\begin{tabular}{|c|c|c|c|c|c|c|c|c|c|c|c|c|c|c|c|}
\hline & \multicolumn{15}{|c|}{ Optimal Sequential Sensor Placement(based on $\left.\operatorname{Tr}\left(P_{d d}\right)\right)$} \\
\hline Meas. Sequence & $\mathbf{1}$ & 2 & 3 & 4 & 5 & 6 & 7 & 8 & 9 & 10 & 11 & 12 & 13 & 14 & 15 \\
\hline Shear Building & 26 & 11 & 17 & 6 & 30 & 21 & 3 & 14 & 10 & 26 & 5 & 12 & 19 & 1 & 28 \\
\hline Frame Building & 28 & 17 & 10 & 22 & 30 & 13 & 23 & 9 & 18 & 29 & 8 & 24 & 15 & 20 & 11 \\
\hline
\end{tabular}

\subsection{Sequential Sensor Placement for Localized Response Estimation}

So far this paper has examined optimal sensor placement based on a global measure such as the trace of the covariance matrix of the state error. If one is interested in determining optimal sensor placement for a specific number of locations it is possible to define cost functions such as 


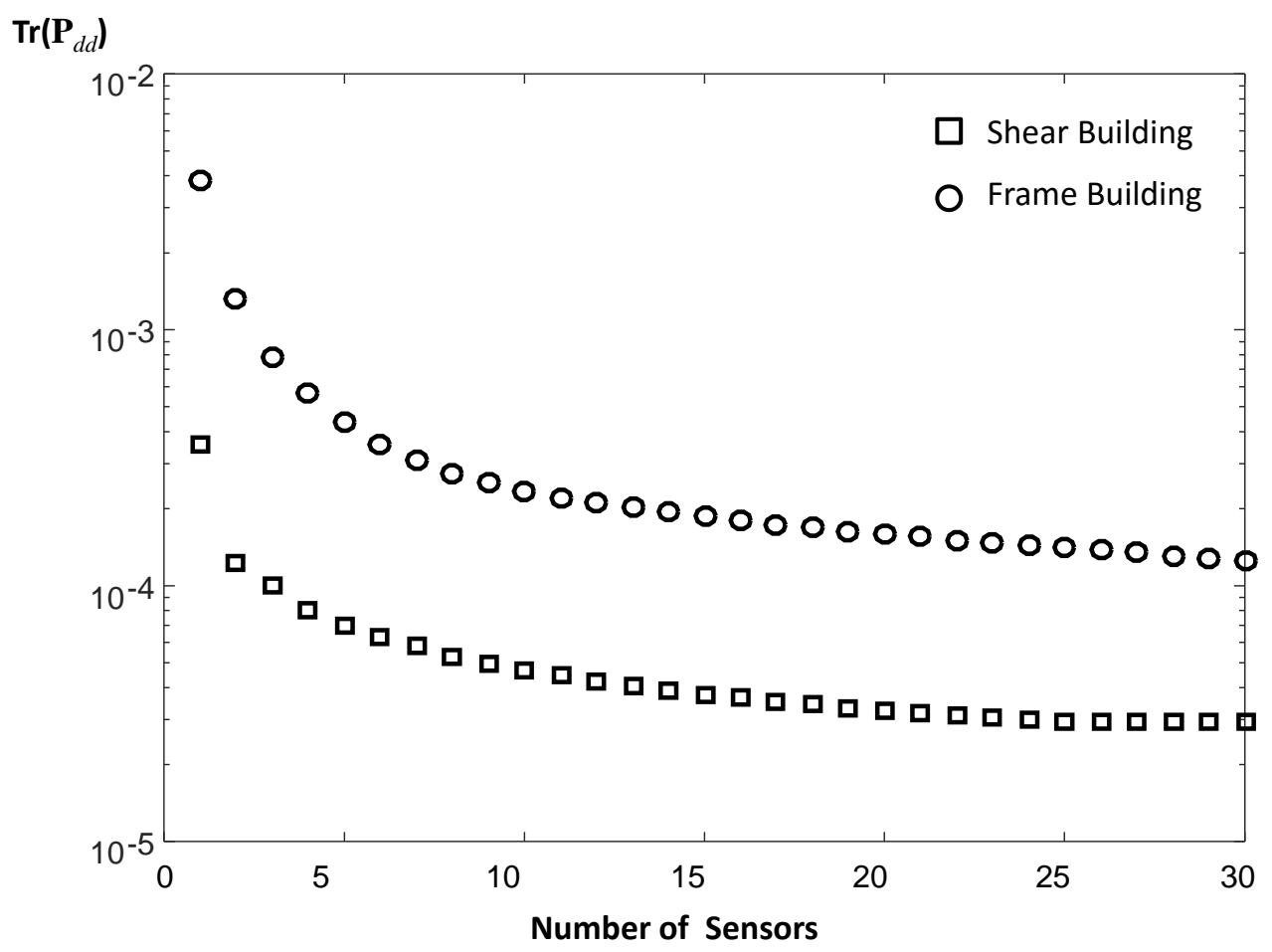

Figure 6: Reduction in the trace of the displacement partition of the state error covariance matrix $\left(\mathbf{P}_{d d}\right)$ as a function of the number of acceleration sensors. Results are shown for two different models, a shear building and a frame building.

$$
J=\sum_{i=1}^{p} \alpha_{i} \sigma_{z_{i}}^{2}
$$

where $\sigma_{z_{i}}$ is the variance of a particular response quantity of interest such as displacement, drift, force, strain, stress, etc. and $\alpha_{i}$ is the weighting factor that indicates the relative importance of estimating $z_{i}$ with respect to all other $z^{\prime} s$. Typically $\sum \alpha_{i}=1$.

Consider as an illustrative example that one is interested in simultaneously computing the drift response between the ground and the first mass (0-1) and the drift between the third mass and the fourth mass (3-4) in the shear building example shown in section 7 . In that case the cost function to minimize would be

$$
J=\alpha_{1} \sigma_{q_{1}}^{2}+\alpha_{2}\left(\sigma_{q_{4}}^{2}+\sigma_{q_{3}}^{2}-2 \sigma_{q_{3}} \sigma_{q_{4}} \rho_{q_{3}, q_{4}}\right)
$$

All the variances necessary to populate this cost function can be obtained directly from the displacement portion of the state error covariance matrix and re-written as

$$
J=\alpha_{1} \mathbf{P}_{1,1}+\alpha_{2}\left(\mathbf{P}_{3,3}+\mathbf{P}_{4,4}-2 \mathbf{P}_{3,4}\right)
$$

It is for the specific user to define the importance factors $\alpha_{1}$ and $\alpha_{2}$. To illustrate the effect of the $\alpha$ factors consider the results shown in Table 5. It shows the optimal sequential accelerometer placement under different relative values of $\alpha_{1}$ and $\alpha_{2}$. As can be seen little difference can be observed, specially for the first few sensors (which are the ones that matter most). 
Table 5: Examining the effect of the importance factor $\alpha$ on optimal sequential accelerometer placement for multiple drift estimation (0-1 and 3-4). Uncorrelated loads and acceleration measurements.

\begin{tabular}{|l||c|c|c|c|c|c|c|c|c|c|c|c|c|c|c|}
\hline \multicolumn{10}{||c|}{} & \multicolumn{11}{|c|}{ Optimal Sequential Sensor Placement } \\
\hline Meas. Sequence & $\mathbf{1}$ & $\mathbf{2}$ & $\mathbf{3}$ & $\mathbf{4}$ & $\mathbf{5}$ & $\mathbf{6}$ & $\mathbf{7}$ & $\mathbf{8}$ & $\mathbf{9}$ & $\mathbf{1 0}$ & $\mathbf{1 1}$ & $\mathbf{1 2}$ & $\mathbf{1 3}$ & $\mathbf{1 4}$ & $\mathbf{1 5}$ \\
\hline$\alpha_{1}=\alpha_{2}$ & 30 & 3 & 1 & 29 & 4 & 27 & 2 & 17 & 5 & 23 & 26 & 7 & 20 & 24 & 8 \\
\hline$\alpha_{1}=2 \alpha_{2}$ & 30 & 1 & 4 & 29 & 3 & 27 & 2 & 17 & 28 & 5 & 25 & 6 & 23 & 26 & 7 \\
\hline $2 \alpha_{1}=\alpha_{2}$ & 30 & 3 & 4 & 29 & 1 & 27 & 5 & 2 & 17 & 28 & 6 & 25 & 26 & 7 & 22 \\
\hline
\end{tabular}

Table 6: Comparison of exhaustive versus sequential optimal sensor placement for estimation of drift 3-4. Uncorrelated loads and displacement measurements.

\begin{tabular}{|l||c|c|c|c|c|}
\hline \multicolumn{1}{|c||}{} & \multicolumn{5}{c|}{ Sensor Placement } \\
\hline Sequential & 6 & 2,6 & $2,4,6$ & $2,4,5,6$ & $2,3,4,5,6$ \\
\hline Variance $\left(\times 10^{-5}\right)$ & 0.80 & 0.66 & 0.58 & 0.53 & 0.47 \\
\hline Comp. Time & 0.7 & 1.2 & 1.7 & 2.3 & 2.8 \\
\hline Optimal & 6 & 2,5 & $2,4,5$ & $2,4,5,6$ & $2,3,4,5,6$ \\
\hline Variance $\left(\times 10^{-5}\right)$ & 0.80 & 0.66 & 0.58 & 0.53 & 0.47 \\
\hline Comp. Time & 0.7 & 8.6 & 98.7 & 560.3 & 2767.1 \\
\hline
\end{tabular}

Table 7: Comparison of exhaustive versus sequential optimal sensor placement for estimation of drift 7-8. Uncorrelated loads and displacement measurements.

\begin{tabular}{|l||c|c|c|c|c|}
\hline \multicolumn{1}{|c||}{} & \multicolumn{5}{c|}{ Optimal Sensor Placement } \\
\hline Sequential & 10 & 6,10 & $6,9,10$ & $6,7,9,10$ & $6,7,8,9,10$ \\
\hline Variance $\left(\times 10^{-5}\right)$ & 0.98 & 0.70 & 0.62 & 0.56 & 0.51 \\
\hline Comp. Time & 0.5 & 1.1 & 1.6 & 2.1 & 2.5 \\
\hline Optimal & 10 & 6,9 & $6,8,9$ & $6,7,9,10$ & $6,7,8,9,10$ \\
\hline Variance $\left(\times 10^{-5}\right)$ & 0.98 & 0.69 & 0.58 & 0.53 & 0.51 \\
\hline Comp. Time & 0.5 & 7.8 & 74.2 & 481.5 & 2964.2 \\
\hline
\end{tabular}

\section{Comparing Sequential Sensor Placement with Exhaustive Search}

Finally, this section presents results aimed at comparing the proposed sequential sensor placement with the absolute optimal sensor placement obtained by carrying out an exhaustive search. All results correspond to the model shown in Fig.2 with noise-contaminated displacement measurements with $\mathbf{R}_{d i s}=(0.0004 m)^{2} \mathbf{I}_{m \times m}$. The loads are niform and uncorrelated with $\mathbf{Q}=\mathbf{I}_{N \times N}$. Table 6 compares the results from the optimal sensor placement (exhausthe search) and the proposed sequential sensor placement algorithm to estimate drift (relative displacement) between

The with minimal computational time. Furthermore, by the fourth sensor and for all practical purposes, both approaches converge. Similar results and trends are obtained regarding optimal sensor placement for estimation of drift 7-8 (See Table 7) and drift 13-14 (see Table 8). These results clearly indicate that the sequential sensor placement proposed in this paper is efficient as it provides a near-optimal solution with a much reduced computational time.

\section{Conclusions}

This paper treats the problem of optimal sequential sensor placement for state estimation in linear structural systems subjected to unmeasured excitations. The criterion for optimal sensor placement was the minimization of 
Table 8: Comparison of exhaustive versus sequential optimal sensor placement for estimation of drift 13-14. Uncorrelated loads and displacement measurements.

\begin{tabular}{|l||c|c|c|c|c|}
\hline \multicolumn{1}{|c||}{} & \multicolumn{5}{c|}{ Optimal Sensor Placement } \\
\hline Sequential & 16 & 12,16 & $12,15,16$ & $12,13,15,16$ & $12,13,14,15,16$ \\
\hline Variance $\left(\times 10^{-5}\right)$ & 1.11 & 0.72 & 0.64 & 0.57 & 0.52 \\
\hline Comp. Time $(\mathrm{s})$ & 0.5 & 1.0 & 1.5 & 2.1 & 2.5 \\
\hline Optimal & 16 & 12,15 & $12,14,15$ & $12,13,14,15$ & $12,13,14,15,16$ \\
\hline Variance $\left(\times 10^{-5}\right)$ & 1.11 & 0.70 & 0.63 & 0.57 & 0.52 \\
\hline Comp. Time $(\mathrm{s})$ & 0.5 & 8.1 & 74.9 & 612.4 & 2751.0 \\
\hline
\end{tabular}

state estimation error variance. Formulas were derived within the context of the Kalman filter.

It was shown that for a system with $n$ possible measurement locations and $m$ measurements, the problem of optimal sensor placement can be efficiently formulated as a sequential problem, thus significantly reducing the computational burden from $\left(\begin{array}{c}n \\ m\end{array}\right)=\frac{n !}{m !(n-m) !}$ Riccati equation evaluations with the exhaustive formulation to $\sum_{j=0}^{m-1}(n-j)$ evaluations with the proposed sequential approach.

Although the sequential sensor placement is not absolutely optimal, it approaches the optimal solution after a few sensors and the computational time is significantly reduced with respect to the exhaustive search. The paper also shows that for the purposes of stress and strain estimation, minimizing the displacement portion of the state error covariance matrix is a better choice than the typical choice of minimizing the trace of full covariance matrix (which includes displacement and velocity).

It was shown that the measurement type (displacement, velocity and acceleration) and spatial correlation of the input have a significant effect in the optimal sensor placement. It was found that small variations resulting from different model classes do not have a significant effect on the optimal sensor placement (so long as the models are reasonable). With regards to using observability as a criterion for optimal sensor placement, it was found that since spatial correlation of the excitation plays a crucial role and observability does not account for the excitation; the exclusive use of observability as a criterion of optimal sensor placement for state estimation is not completely adequate.

Finally, and not surprisingly, it was found that increasing the number of sensors beyond a certain point results in marginal information gain (uncertainty reduction) i.e. there is a point after which little estimation accuracy is gained by adding more sensors. This points to the need of using measurements more efficiently as opposed to simply placing more sensors on structures for monitoring.

\section{Acknowledgements}

The research presented in this paper was partially supported by the National Science Foundation (NSF) awards ECC-1342190 and CMMI-1453502. NSF support is gratefully acknowledged. The authors would also like to thank the anonymous reviewers for their suggestions which improved the overall quality of the paper.

\section{References}

[1] C Papadimitriou, CP Fritzen, P Kraemer, and E Ntotsios. Fatigue predictions in entire body of metallic structures from a limited number of vibration sensors using Kalman filtering. J Structural Control and Health Monitoring, 18(5):554 - 573, 2011.

[2] Erazo, K. and Hernandez, E.M. Tracking Stress Response in Structures Using Acceleration Measurements: Experimental Validation Mechanical Systems and Signal Processing, 43(12): 41-152, 2014

[3] Carmichael D.G., 1979. The state estimation problem in experimental structural mechanics. Procedings of the Third International Conference on Applications of Statistics and Probability in Soil and Structural Engineering.

[4] Waller H. and Schmidt R. 1990. The application of state estimation of state observers in structural dynamics. Mechanical Systems and Signal Proccesing 4 195-213.

[5] H.Waller and R. Schmidt. The application of state observers in structural dynamics. Mechanical Systems and Signal Processing, 4(3):195 213, 1990.

[6] Wilde P. and Kozakiewicz A., 1993. Kalman Filter in the Analysis of Vibrations Due to Water Waves, Advanced Series in Ocean Engineering, World Scientific. River Edge, N.J. 
[7] Hernandez, E.M. and Bernal, D. (2008) State Estimation in Structural Systems with Uncertain Stiffness and Damping Matrices ASCE Journal of Engineering Mechanics, (134)3:252-258

[8] EM Hernandez. A natural observer for optimal state estimation in second order linear structural systems. Mechanical Systems and Signal Processing, 25:2938 - 2947, 2011.

[9] EM Hernandez. Optimal model-based state estimation in mechanical and structural systems. J Structural Control Health Monitoring, 20:532 $-543,2011$

[10] EM Hernandez, D Bernal and L. Caracoglia. On-line Monitoring of Wind-Induced Stresses in Instrumented Structures. J Struct Control and Health Mon., 20(10):1291-1301, 2013.

[11] K Maes, A Iliopoulus, W Weijtjens, C Devriendt and G Lombaert. Dynamic strain estimation for fatigue assessment of an offshore monopile wind turbine using filtering and modal expansion algorithms. J Mech. Sys. and Signal Proc., 76-77:592-611, 2016.

[12] RE Kalman. A new approach to linear filtering and prediction problem. J Basic Engineering, Trans ASME, 82:35 - 45, 1960

[13] A. Gelb (Editor) Applied Optimal Estimation. MIT Press, Cambridge, USA, 1996.

[14] M Grewal and A Andrews. Kalman Filtering, Theory and Practice using MATLAB. Wiley, New Jersey, USA, 1998.

[15] EM Hernandez and NR Polanco. A lower bound for the variance of frequency and damping ratio identified from noisy vibration measurements. Journal of Structural Control and Health Monitoring. DOI: 10.1002/stc.1757

[16] C. Papadimitriou. Optimal sensor placement methodology for parameter identification of structural systems. Journal of Sound and Vibration, 278:923 - 947, 2004.

[17] DC Krammer. Optimal sensor placement for modal identification using system-realization methods. J of Guidance, Control and Dynamics, 19(3):729-731, 1996.

[18] K-V Yuen and S-C Kuok. Efficient Bayesian sensor placement algorithm for structural identification: a general approach for multi-type sensory systems. Earthquake Eng. anf Struct. Dyn, 44:757-774

[19] GW van den Linder, A Emami, RL Kosut, H. Sedarat, JP Lynch. Near-Optimal Sensor Placement for Health Monitoring of Civil Structures. American Control Conference, 2011. San Francisco, CA.

[20] Weickgenannt M., Neuhaeuser S., Henke B, Sobek W., Sawodny O., 2013. Optimal sensor placement for state estimation of a thin doublecurved shell structure. Mechatronics, 23, pp. 346-354.

[21] Zhang C.D., Xu Y.L., 2016. Optimal multi-type sensor placement for response and excitation reconstruction. Journal of Sound and Vibration, 360, pp. 112-128

[22] W Brogan. Modern Control Theory. Prentice Hall, New Jersey, USA, 1991. 